\title{
Mortalidade perinatal em duas coortes de base populacional no Sul do Brasil: tendências e diferenciais
}

Ana M. B. Menezes 1 Fernando C. Barros ${ }^{2}$ Cesar G. Victora ${ }^{2}$ Clarita Alves 1 Cristina Rocha 1

Elaine Albernaz 3 Flávio S. Menezes 4 Heitor A. Jannke 4

\author{
Perinatal mortality in two population-based \\ cohorts from southern Brazil: trends and \\ differences
}

1 Departamento de Clínica Médica.

2 Departamento de Medicina Social.

3 Departamento MaternoInfantil

4 Departamento de Cirurgia Geral. Faculdade de Medicina, Universidade Federal de Pelotas 464, Pelotas, RS 96001-970, Brasil.

\begin{abstract}
Trends in perinatal mortality were studied in Pelotas (southern Brazil) through surveys carried out in 1982 and 1993. All hospital births and perinatal deaths were assessed by daily visits to all maternity hospitals. Cause of death was determined through review of hospital case notes, interviews with pediatricians, and autopsies. The perinatal mortality rate decreased by $31 \%$ over the decade. Under-recording of perinatal deaths was reduced from 42.1\% in 1982 to 6.8\% in 1993. A reduction of 58\% in antepartum fetal deaths was observed in 1993 (47\% for deaths due to immaturity and 62\% for other causes). The rate of deaths due to asphyxia increased from 4.5/1.000 in 1982 to 8.3/1.000 in 1993. Female babies showed a significantly lower perinatal mortality rate than males. Decreases in perinatal mortality rates in 1993 were observed in virtually all birthweight and family income groups. The most important reduction (68\%) was observed for low birthweight babies belonging to high-income families. As in 1982, preterm babies were three times more likely to die in the perinatal period than babies with intrauterine growth retardation. Despite major decreases in perinatal mortality over the decade, large gaps between different social groups persisted.
\end{abstract}

Key words Perinatal Mortality; Mortality; Birthweight; Child Health; Epidemiology

Resumo A evolução da mortalidade perinatal foi estudada em Pelotas, Rio Grande do Sul, através de dois estudos de coorte realizados em 1982 e 1993. Todos os nascimentos hospitalares e os óbitos foram monitorizados com visitas diárias aos hospitais. A causa da morte foi determinada através de informações do prontuário, entrevista com o pediatra e de necrópsias. O coeficiente de mortalidade perinatal sofreu uma redução de $31 \%$ na década. O sub-registro que era de 42,1\% em 1982, foi de apenas 6,8\% em 1993. Dentre as causas de mortalidade perinatal, houve uma redução em 1993 de 58\% para óbitos fetais antepartum, 47\% para imaturidade e 62\% para outras causas. Os coeficientes para asfixia sofreram um aumento de 4,5/1.000 para 8,3/1.000. As meninas apresentaram um coeficiente de mortalidade perinatal menor do que os meninos. Os coeficientes de mortalidade perinatal conforme peso ao nascer e renda familiar sofreram importantes reduções, sendo que a mais notável foi para crianças de baixo peso e de famílias de renda alta, com uma queda de 68\%. Assim como em 1982, os recém-nascidos pré-termo tiveram coeficientes três vezes mais elevados do que crianças pequenas para a idade gestacional. Apesar da queda dos índices de mortalidade na década, as diferenças entre os grupos sociais mantiveramse grandes.

Palavras-chave Mortalidade Perinatal; Mortalidade; Peso ao Nascer; Saúde da Criança; Epidemiologia 


\section{Introdução}

Em regiões onde os problemas mais fundamentais de sobrevivência, como condições de moradia e saneamento, encontram-se mais bem equacionados, as causas perinatais são as principais responsáveis pela mortalidade infantil, pois há uma redução dos óbitos causados por diarréias e infecções respiratórias, causas mais importantes de mortalidade em países subdesenvolvidos. No Rio Grande do Sul, dados de 1993 mostram que $46 \%$ dos óbitos infantis são de causa perinatal, sendo seguidos de anomalias congênitas (16\%) e infecções respiratórias (15\%) (DIS/ATMA/RS, 1995). Portanto, especialmente em regiões neste estágio de desenvolvimento, o estudo detalhado das patologias perinatais é de fundamental importância para a prevenção de óbitos infantis.

$O$ estudo dos coeficientes de mortalidade perinatal, da mesma forma como da mortalidade materna, também é muito útil para a avaliação da qualidade da atenção obstétrica e pediátrica oferecida nos períodos pré-natal e perinatal (Cartilidge \& Stewart, 1995). Para que esta informação seja confiável, é necessário que o registro dos óbitos seja adequado, o que não é observado em muitos locais. No estudo perinatal realizado em Pelotas, em 1982, foi detectado um elevado percentual de sub-registro de óbitos perinatais, principalmente às custas de óbitos fetais, atingindo 47\% (Barros et al., 1985). Outro aspecto a ser considerado nas comparações de coeficientes de mortalidade perinatal refere-se aos critérios de inclusão conforme a idade gestacional e o peso de nascimento (Cartilidge \& Stewart, 1995). Estudos recentes tendem a incluir óbitos fetais a partir de 24 semanas de gestação nos registros de mortalidade perinatal, enquanto que o critério clássico da Organização Mundial da Saúde recomenda apenas a inclusão de crianças com peso ao nascer igual ou superior a 500 gramas, tanto para nascidos vivos como para natimortos (Cartilidge \& Stewart, 1995).

Este trabalho tem a finalidade de comparar a evolução da mortalidade perinatal ocorrida entre 1982 (Victora et al., 1989) e 1993 na cidade de Pelotas. Através da comparação dos indicadores de saúde perinatal nestes anos pretende-se contribuir para o melhor entendimento dos fenômenos médicos e sociais ocorridos no período, com vistas a propor medidas preventivas mais adequadas.

\section{Metodologia}

Todos os nascimentos ocorridos em Pelotas em 1993 foram acompanhados através de um estudo que utilizou metodologia semelhante ao realizado em 1982 (Victora et al., 1996). Foram realizadas visitas diárias às cinco maternidades da cidade através das quais foram constatados 5.304 nascimentos. Todas as mães foram entrevistadas, por ocasião do nascimento da criança, com o auxílio de um questionário que contemplava, entre outros, temas demográficos e sócio-econômicos, a história reprodutiva e a utilização de serviços de saúde. Informações mais detalhadas da metodologia do estudo longitudinal estão descritas em outras publicações (Victora et al., 1996; Victora et al., 1996).

Neste artigo, a metodologia apresentada diz respeito ao subestudo de mortalidade perinatal. Todos os óbitos perinatais (óbitos fetais - a partir da 28a semana de gestação e neonatais precoces - ocorridos na primeira semana de vida) foram monitorizados em Pelotas no período de 1o de janeiro de 1993 até os primeiros sete dias de 1994. Todas as maternidades e as três Unidades de Tratamento Intensivo dos hospitais da cidade foram visitadas diariamente. Além disso, quinzenalmente, os berçários, enfermarias pediátricas e os serviços de Pronto Socorro também eram visitados. Através de visitas mensais aos cartórios, cemitérios e à Delegacia Regional de Saúde, foram identificados óbitos extra-hospitalares.

Para detectar a causa básica do óbito fetal e o momento do mesmo (antepartum ou intrapartum), foi realizada uma entrevista com o obstetra responsável pelo parto, e este opinava sobre a causa básica da morte e o momento do óbito fetal. Também eram coletadas algumas informações específicas, tais como: ocorrência de descolamento de placenta, prolapso de cordão, trabalho de parto pré-termo espontâneo, apresentação pélvica, placenta prévia, doença hipertensiva específica da gravidez, incompetência de colo uterino, ruptura uterina, doenças infecciosas pélvicas, hipertensão arterial sistêmica e outras doenças.

Para óbitos neonatais precoces, era realizada uma entrevista com o pediatra e fazia-se uma revisão sistemática do prontuário, no caso de crianças hospitalizadas.

Tentou-se obter uma autorização dos pais para a realização de necrópsias de todos os óbitos ocorridos após o nascimento. A solicitação era feita pelo pediatra que havia atendido a criança e, com a concordância dos pais, a necrópsia era realizada. Os exames post-mortem foram feitos diariamente pelo Laboratório de 
Patologia da Faculdade de Medicina, sendo que o transporte do corpo era feito pela funerária da Prefeitura Municipal.

A causa básica da morte era determinada por dois médicos que funcionavam como árbitros independentes. Estes, sem conhecer o atestado de óbito oficial obtido na Secretaria da Saúde, completavam dois outros atestados de óbito, com base nas informações colhidas sobre o caso. Havendo discordância entre os dois árbitros, era solicitado a um terceiro árbitro opinar sobre o diagnóstico final do caso. Seguindo a mesma metodologia de 1982, foi utilizada a classificação proposta por Wigglesworth (Wigglesworth, 1980; Keeling, 1989), segundo a qual as causas de morte são reunidas em cinco grupos: 1) mortes fetais antepartum, 2) malformações congênitas, 3) imaturidade, 4) asfixia, 5) outras causas de óbitos fetais ou neonatais precoces. A codificação dos atestados de óbito (Nona Classificação Internacional de Doenças) (OMS, 1980) foi realizada pelo coordenador da Divisão de Informação em Saúde da Secretaria da Saúde e do Meio Ambiente do Rio Grande do Sul.

Não foram incluídos na análise dois óbitos fetais e um óbito neonatal precoce, por recusa das famílias em responder a entrevista perinatal.

Os desfechos estudados foram o coeficiente de mortalidade perinatal (número de óbitos fetais e neonatais precoces por 1.000 nascimentos vivos e mortos), com os seus dois componentes: coeficiente de mortalidade fetal (número de óbitos fetais por 1.000 nascimentos vivos e mortos) e coeficiente de mortalidade neonatal precoce (número de óbitos na primeira semana de vida por 1.000 nascidos vivos). As variáveis independentes incluídas no presente artigo foram: sexo, peso ao nascer em gramas, renda familiar expressa em salários mínimos, idade gestacional calculada através da data da última menstruação, número de consultas prénatais e tipo de parto (vaginal ou cesariana). $\mathrm{O}$ teste do Qui-Quadrado foi utilizado para testar associação entre as variáveis independentes e os coeficientes de mortalidade.

\section{Resultados}

Houve uma redução no número de nascimentos durante a década, de 6.011 em 1982 para 5.304 em 1993. Enquanto em 1982 ocorreram 194 óbitos perinatais, dos quais 97 foram óbitos fetais e 97 neonatais precoces, em 1993 os óbitos perinatais foram reduzidos para 117 , sendo 55 no período fetal e 62 no período neo- natal precoce (Tabela 1). A Tabela 1 mostra, portanto, que houve uma importante redução de $31 \%$ no coeficiente de mortalidade perinatal - de 32,2/1.000 nascimentos em 1982 para $22,1 / 1.000$ nascimentos em 1993. Esta diminuição da mortalidade foi observada igualmente no período fetal e no neonatal precoce.

Com relação aos óbitos fetais, a queda da mortalidade em 1993 deveu-se mais à redução dos óbitos ocorridos antes do início do trabalho de parto, com um coeficiente de 7,4/1.000, comparado a 14,9/1.000 em 1982. Os índices de mortalidade fetal intrapartum permaneceram inalterados. Quanto aos óbitos neonatais precoces, a maior redução da mortalidade (45\%) foi observada no primeiro dia de vida.

Dos óbitos neonatais detectados, apenas um ocorreu no domicílio; quatro óbitos neonatais precoces aconteceram nos berçários, tendo os demais ocorrido nas UTIs pediátricas.

Em relação às necrópsias solicitadas, o percentual alcançado para os óbitos neonatais precoces foi de $31,7 \%$.

Uma diferença importante encontrada entre 1982 e 1993 foi o sub-registro de óbitos perinatais encontrado. Enquanto em 1982 este índice atingiu a cifra de $42,1 \%$, o mesmo foi de apenas $6,8 \%$ em 1993 , sendo $7,3 \%$ para óbitos fetais e $6,5 \%$ para óbitos neonatais precoces.

A Tabela 2 mostra as causas dos óbitos perinatais, segundo a classificação de Wigglesworth (Wigglesworth, 1980; Keeling, et al.,

Tabela 1

Mortalidade perinatal em Pelotas: 1982-1993.

\begin{tabular}{lcc}
\hline Indicadores & 1982 & 1993 \\
& $n$ & $n$ \\
\hline Número de Nascimentos & 6.011 & 5.304 \\
Óbitos Fetais & & \\
Número & 97 & 10,5 \\
Coeficiente por mil & 16,1 & $(47 \%)$ \\
\% dos óbitos perinatais & $(50 \%)$ & \\
& & 62 \\
Óbitos Neonatais Precoces & 97 & 11,7 \\
Número & 16,4 & $(53 \%)$ \\
Coeficiente por mil & $(50 \%)$ & \\
\% dos óbitos perinatais & & 117 \\
Óbitos Perinatais & 194 & 22,1 \\
Número & 32,2 & $(100 \%)$ \\
Coeficiente por mil & $(100 \%)$ & \\
\% dos óbitos perinatais & & \\
\hline
\end{tabular}


Coeficientes de mortalidade perinatal (por mil) conforme a classificação de Wigglesworth. Pelotas: 1982-1993.

\begin{tabular}{|c|c|c|c|c|c|c|}
\hline \multirow[t]{3}{*}{ Causas } & \multicolumn{6}{|c|}{ Coeficientes de mortalidade } \\
\hline & 82 & 93 & 82 & 93 & 82 & 93 \\
\hline & $\mathrm{n}$ & $\mathrm{n}$ & & $\%$ & Coefic. & Coefic. \\
\hline Natimorto antepartum & 79 & 29 & 41 & 25 & 13,1 & 5,5 \\
\hline Malformações & 14 & 13 & 7 & 11 & 2,3 & 2,5 \\
\hline Imaturidade & 45 & 21 & 23 & 17 & 7,3 & 3,9 \\
\hline Asfixia & 27 & 44 & 14 & 38 & 4,5 & 8,3 \\
\hline Outras causas & 29 & 10 & 15 & 8 & 5,0 & 1,9 \\
\hline Todas as causas & 194 & 117 & 100 & 100 & 32,3 & 22,1 \\
\hline
\end{tabular}

Coefic.: coeficiente

1989). Entre 1982 e 1993, houve uma redução dos coeficientes de mortalidade de $58 \%$ para óbitos fetais antepartum, $47 \%$ para imaturidade e $62 \%$ para outras causas. Quanto às malformações congênitas, os coeficientes mantiveram-se os mesmos, sendo que para os óbitos por asfixia observou-se um aumento de 4,5/1.000 em 1982 para 8,3/1.000 em 1993.

No que se refere à mortalidade conforme o sexo dos recém-nascidos, ao passo que, em 1982, não foi observada nenhuma diferença nos coeficientes, no ano de 1993, o coeficiente de mortalidade perinatal foi significativamente menor entre as crianças de sexo feminino 15,6/1.000, em comparação com 23,8/1.000 entre os meninos (Tabela 3). Esta diferença deveu-se ao componente neonatal precoce, em que a mortalidade feminina foi a metade daquela observada entre os meninos.

A Tabela 4 mostra os coeficientes de mortalidade perinatal, fetal e neonatal precoce em 1982 e 1993, conforme o peso ao nascer. Para a mortalidade fetal, notam-se reduções importantes nos coeficientes de 1993 em todos os grupos de peso. Já com relação ao componente neonatal precoce da mortalidade, houve um surpreendente aumento nos coeficientes para crianças na faixa de 1.000 a 2.000 gramas. Ao ser analisada a mortalidade, com o peso ao nascer dicotomizado em baixo peso (menor que 2.500 g) e peso igual ou superior a $2.500 \mathrm{~g}$, observase uma queda importante dos coeficientes de mortalidade perinatal de 1982 para 1993 nos dois grupos de peso (Tabela 4). No grupo de crianças de baixo peso ao nascer, a mortalidade foi reduzida à metade - de 203,4/1.000 para $106,7 / 1.000$. Nas crianças com peso igual ou superior a $2.500 \mathrm{~g}$, este coeficiente, em 1993, foi de 12,9/1.000, tendo sido reduzido 2,8 vezes, em 1993, para 4,6/1.000. Portanto, as quedas de mortalidade conforme o peso ao nascer, na década, foram bastante significativas.

A relação entre a mortalidade perinatal e a renda familiar é analisada na Figura 1. Na população de 1993, as crianças de famílias de menor renda (até um salário mínimo de renda familiar mensal) tiveram uma mortalidade 6 vezes maior do que aquelas pertencentes a famílias com renda superior a 10 salários mínimos. Note-se que, em 1982, esta diferença havia si-

Tabela 3

Coeficientes de mortalidade fetal, neonatal precoce e perinatal (por mil) conforme sexo. Pelotas: 1982-1993.

\begin{tabular}{lcccccc}
\hline Variável & \multicolumn{2}{c}{ Fetal } & \multicolumn{2}{c}{ Neonatal Precoce } & \multicolumn{2}{c}{ Perinatal } \\
& 82 & 93 & 82 & 93 & 82 & 93 \\
\hline $\begin{array}{l}\text { Gênero } \\
\text { masculino } \\
\text { feminino }\end{array}$ & 15,9 & 8,8 & 16,5 & 15,1 & 32,1 & 23,8 \\
& 16,1 & 7,4 & 15,3 & 8,2 & 31,1 & 15,6 \\
$P^{*}$ & $>0,05$ & $>0,05$ & $>0,05$ & $<0,05$ & $>0,05$ & $<0,05$ \\
\hline
\end{tabular}

* teste $\chi^{2}$ 
Coeficientes de mortalidade fetal, neonatal precoce e perinatal (por mil) conforme peso ao nascer: 1982-1993

\begin{tabular}{|c|c|c|c|c|c|c|}
\hline \multirow[t]{3}{*}{ Peso ao nascer (g) } & \multicolumn{6}{|c|}{ Coeficientes de Mortalidade } \\
\hline & \multicolumn{2}{|c|}{ Fetal } & \multicolumn{2}{|c|}{ Neonatal Precoce } & \multicolumn{2}{|c|}{ Perinatal } \\
\hline & 82 & 93 & 82 & 93 & 82 & 93 \\
\hline$<1.000$ & (a) & 238,1 & 952,3 & 588,2 & 952,3 & 714,3 \\
\hline $1.000-1.499$ & 306,4 & 117,6 & 488,3 & 500,0 & 645 & 1558,8 \\
\hline $1.500-1.999$ & 128,0 & 22,2 & 64,2 & 136,4 & 184,0 & 155,6 \\
\hline $2.000-2.499$ & 42,4 & 10,5 & 55,4 & 10,6 & 95,4 & 21,1 \\
\hline $2.500-2.999$ & 9,9 & 0,8 & 7,1 & 3,8 & 17,0 & 4,6 \\
\hline $3.000-3.499$ & 6,2 & 3,4 & 4,9 & 2,0 & 11,1 & 5,3 \\
\hline $3.500-3.999$ & 6,3 & 1,8 & 2,8 & 0,9 & 9,1 & 2,8 \\
\hline$\geq 4.000$ & 19,8 & 3,6 & 2,8 & 3,6 & 22,7 & 7,1 \\
\hline$p^{*}$ & $<0,001$ & $<0,001$ & $<0,001$ & $<0,001$ & $<0,001$ & $<0,001$ \\
\hline$<2.500$ & 87,1 & 28,6 & 127,3 & 80,2 & 203,4 & 106,7 \\
\hline$\leq 2.500$ & 8,1 & 2,3 & 4,8 & 2,3 & 12,9 & 4,6 \\
\hline$p^{*}$ & $<0,001$ & $<0,001$ & $<0,001$ & $<0,001$ & $<0,001$ & $<0,001$ \\
\hline Todos & 16,1 & 10,4 & 16,4 & 11,7 & 32,2 & 22,1 \\
\hline
\end{tabular}

(a) Natimortos com menos de $1.000 \mathrm{~g}$ foram excluídos do estudo * teste $\chi^{2}$

do de quatro vezes. Portanto, embora tenha ocorrido durante a década uma queda nos coeficientes de mortalidade em todos os grupos de renda, a redução mais importante ocorreu entre crianças de maior renda - de 12/1.000 em 1982 para 5/1.000 em 1993.

A Tabela 5 revela os coeficientes de mortalidade perinatal, fetal e neonatal precoce, em 1982 e 1993, conforme o peso ao nascer e a idade gestacional, calculada pela data da última menstruação. Percebe-se que, em todos os grupos, ocorreram importantes reduções durante a década. Os recém-nascidos pré-termo permaneceram, como já ocorria em 1982, apresentando coeficientes de mortalidade perinatal três vezes mais elevados do que as crianças pequenas para a idade gestacional.

A Tabela 6 mostra que as taxas de mortalidade não sofreram qualquer alteração quanto ao tipo de parto em 1993, embora em 1982 tenha sido observada uma taxa de mortalidade neonatal precoce maior entre crianças nascidas de cesariana. No que diz respeito a consultas pré-natais, as informações de 1993 confirmam o que já havia sido constatado em 1982: crianças cujas mães consultaram mais de cinco vezes durante a gestação apresentaram coeficientes de mortalidade significativamente menores. Esta informação deve ser tratada com a devida cautela, tendo em vista que crianças nascidas pré-termo, que são as que apresentam a maior mortalidade, são aquelas cujas mães tiveram menos chances de terem o número adequado de consultas.

A relação entre mortalidade perinatal e renda familiar, analisada juntamente com peso ao nascer, em 1982 e 1993, é demonstrada na Figura 2. No grupo de crianças de baixo peso ao nascer pertencentes a famílias de baixa renda (menos de três salários mínimos mensais), observa-se uma redução da mortalidade perinatal de 38\% - 204/1.000 para 126/1.000. A redução na década é mais notável para as crianças

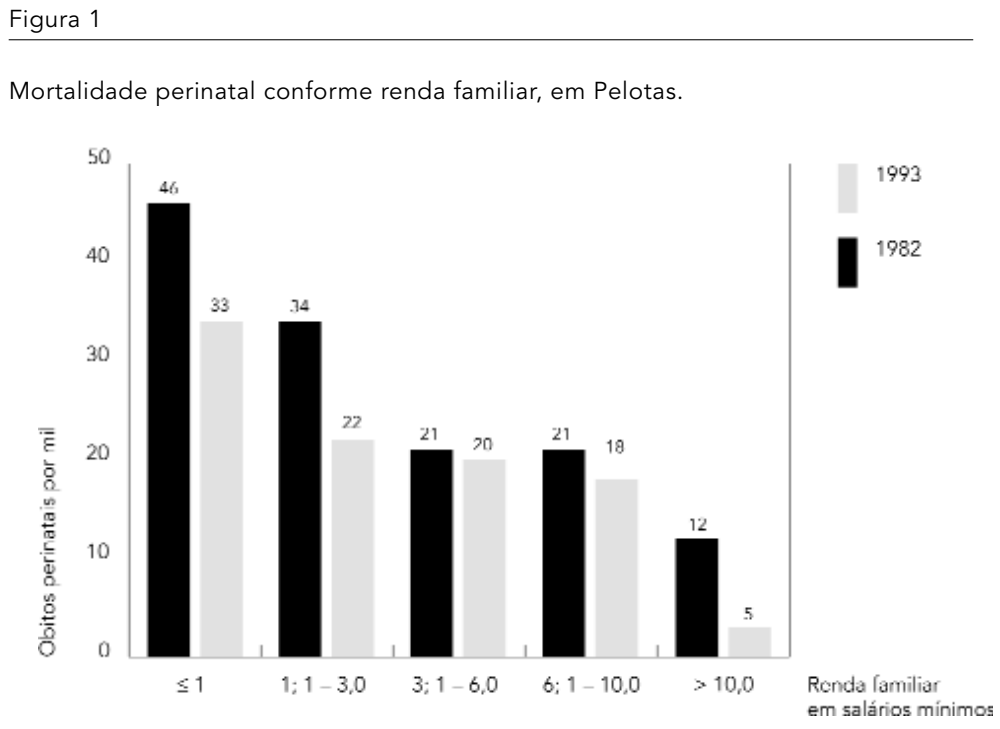


Coeficientes de mortalidade fetal, neonatal precoce e perinatal (por mil) conforme o peso ao nascer e a idade gestacional (data da última menstruação). Pelotas: 1982-1993.

\begin{tabular}{|c|c|c|c|c|c|c|c|c|}
\hline \multirow{3}{*}{$\begin{array}{l}\text { Peso ao nascer e } \\
\text { idade gestacional }\end{array}$} & \multicolumn{6}{|c|}{ Coeficientes de Mortalidade } & \multicolumn{2}{|c|}{ Óbitos/Nascimentos } \\
\hline & \multicolumn{2}{|c|}{ Fetal } & \multicolumn{2}{|c|}{ Neonatal Precoce } & \multicolumn{2}{|c|}{ Perinatal } & \multirow[b]{2}{*}{82} & \multirow[b]{2}{*}{93} \\
\hline & 82 & 93 & 82 & 93 & 82 & 93 & & \\
\hline $\begin{array}{l}\text { Pequenas para a } \\
\text { idade gestacional }\end{array}$ & 41 & 5 & 20 & 10 & 61 & 15 & $44 / 723$ & $12 / 783$ \\
\hline \multicolumn{9}{|l|}{$\begin{array}{l}\text { Peso adequado para } \\
\text { a idade gestacional: }\end{array}$} \\
\hline Pré-termo & 59 & 10 & 75 & 36 & 129 & 45 & $35 / 271$ & $23 / 510$ \\
\hline A termo & 6 & 1 & 4 & 1 & 11 & 3 & $40 / 3.742$ & $9 / 3.380$ \\
\hline$p^{*}$ & $>0,001$ & $<0,003$ & $<0,001$ & $<0,001$ & $<0,001$ & $<0,001$ & & \\
\hline
\end{tabular}

* teste $\chi^{2}$

Tabela 6

Coeficientes de mortalidade fetal, neonatal precoce e perinatal (por mil) conforme o número de consultas pré-natais e o tipo de parto. Pelotas: 1982-1993.

\begin{tabular}{|c|c|c|c|c|c|c|}
\hline \multirow[t]{2}{*}{ Variáveis } & \multicolumn{2}{|c|}{ Fetal } & \multicolumn{2}{|c|}{ Neonatal Precoce } & \multicolumn{2}{|c|}{ Perinatal } \\
\hline & 82 & 93 & 82 & 93 & 82 & 93 \\
\hline \multicolumn{7}{|c|}{ Consultas pré-natais } \\
\hline Nenhuma & 22,8 & 23,3 & 40,1 & 27,9 & 62,0 & 50,6 \\
\hline $1-4$ & 24,7 & 24,8 & 23,4 & 26,9 & 47,6 & 51,0 \\
\hline $5-9$ & 14,8 & 7,4 & 13,9 & 8,5 & 28,5 & 15,8 \\
\hline$\geq 10$ & 6,8 & 6,7 & 10,4 & 8,7 & 17,2 & 15,4 \\
\hline$p^{\star}$ & 0,01 & $<0,001$ & $<0,001$ & $<0,001$ & $<0,001$ & $<0,001$ \\
\hline \multicolumn{7}{|l|}{ Tipo de parto } \\
\hline Vaginal & 16,0 & 10,0 & 14,0 & 11,2 & 29,8 & 21,2 \\
\hline Cesariana & 16,2 & 11,1 & 22,6 & 13,1 & 38,5 & 24,1 \\
\hline$p^{*}$ & $>0,05$ & $>0,05$ & $<0,05$ & $>0,05$ & $>0,05$ & $>0,05$ \\
\hline
\end{tabular}

* teste $\chi^{2}$

de baixo peso, de famílias de renda mais alta, caso em que a queda é de 68\% - 182/1.000 e 58/1.000, respectivamente.

Ainda em relação à Figura 2, chama a atenção que, no ano de 1993, a mortalidade entre crianças de peso adequado foi igual, independentemente da renda familiar, enquanto que, para crianças de baixo peso ao nascer, a mortalidade perinatal foi de 126/1.000 no grupo de baixa renda e de 58/1.000 para as crianças cujas famílias percebiam mais de três salários mínimos mensais. Além disso, esta diferença nos coeficientes de mortalidade perinatal entre crianças de baixo peso foi devida unicamente ao componente neonatal precoce, cujas cifras foram de 102/1.000 entre as crianças mais po- bres e 30/1.000 entre as crianças de famílias de maior renda (Figura 3).

\section{Discussão}

Os problemas perinatais foram a principal causa de mortalidade infantil em Pelotas em 1993, tendo sido responsáveis por $52 \%$ dos óbitos ocorridos no primeiro ano de vida (Menezes et al., 1996). Justifica-se, portanto, estudar em profundidade as razões para as mortes neste grupo, com vistas a propor medidas preventivas. Os estudos de base populacional de $1982 \mathrm{e}$ 1993 permitiram, também, uma avaliação da evolução temporal da mortalidade perinatal na 
década, com uma análise detalhada dos fatores que determinaram as mudanças destes indicadores.

Uma das limitações no estudo dos coeficientes de mortalidade perinatal advém da má qualidade dos registros de óbitos, tanto no que se refere ao sub-registro, como aos diferentes critérios utilizados para o registro. O presente estudo monitorizou os óbitos através do cruzamento de diversas fontes de informação, o que sugere que praticamente todos os óbitos foram detectados. Um importante achado foi a grande redução de sub-registro de 1982 (42,1\%) para 1993 (6,8\%) (Barros et al., 1985). A principal razão para esta importante diminuição do subregistro de óbitos foi a divulgação e discussão dos dados de sub-registro de 1982 com os profissionais de saúde, especialmente com as equipes da Secretaria da Saúde responsáveis pela monitorização destes óbitos. Quanto à segunda limitação sobre os diferentes critérios de inclusão utilizados nos registros de mortalidade perinatal, a idade gestacional de 28 semanas e peso acima de 500 gramas foram os critérios de inclusão adotados para o registro dos natimortos neste estudo, o que permitiu a comparação com o de 1982. Recentemente vem sendo discutido na literatura que os óbitos por condições perinatais potencialmente tratáveis devem ser incluídos nos coeficientes de mortalidade perinatal, e aqueles considerados inevitáveis, excluídos (Cartlidge \& Stewart, 1995).

A redução dos coeficientes de mortalidade perinatal - de 32,2/1.000 em 1982 para 22,1/ 1.000 em 1993 - foi bastante expressiva: $31 \%$. Esta redução é superior à descrita para a última década no Estado do Rio Grande do Sul, que foi de 23\% (DIS/ATMA/RS, 1995). Cabe ressaltar que a diminuição da mortalidade ocorreu igualmente às custas dos componentes fetal e neonatal precoce.

Quando se procura analisar reduções de mortalidade perinatal e infantil, deve-se levar em consideração possíveis modificações das condições maternas, como idade e paridade (Morris et al., 1975), que podem influenciar a distribuição do peso ao nascer, que é, por sua vez, o principal determinante da sobrevivência infantil. Em outros artigos desta série (Horta et al., 1996; Tomasi et al., 1996), pode ser observado que as condições maternas, no que se refere à idade e paridade, não experimentaram modificações importantes na década, e que a prevalência de baixo peso ao nascer aumentou de 9,0\% em 1982 para 9,9\% em 1993. Portanto, as reduções da mortalidade perinatal não podem ser atribuídas a estes fatores. Outras possíveis explicações para essas quedas são melhorias
Figura 2

Mortalidade perinatal conforme renda e peso ao nascer, em Pelotas.

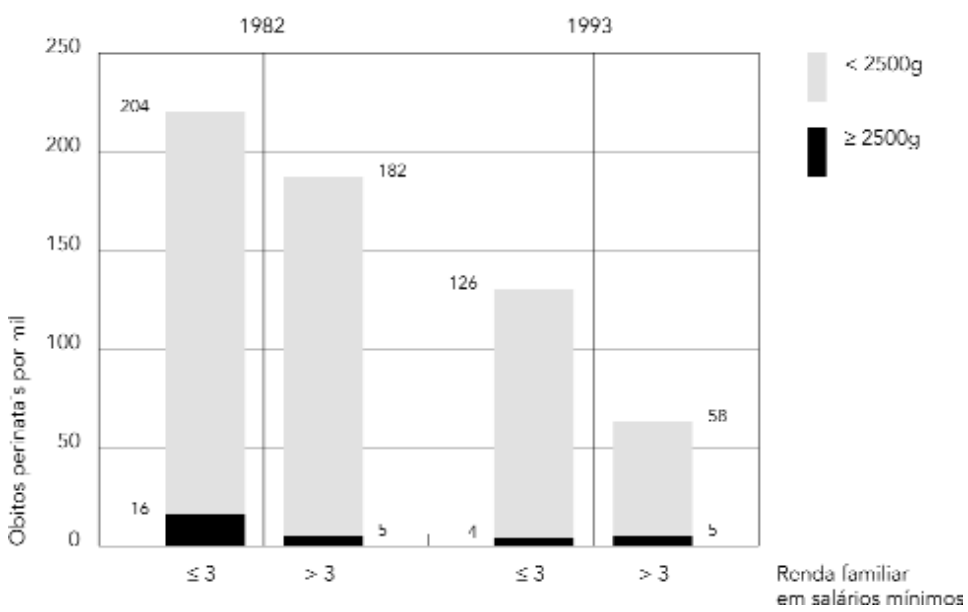

Figura 3

Mortalidade fetal e neonatal precoce conforme renda e peso ao nascer. Pelotas, 1993.

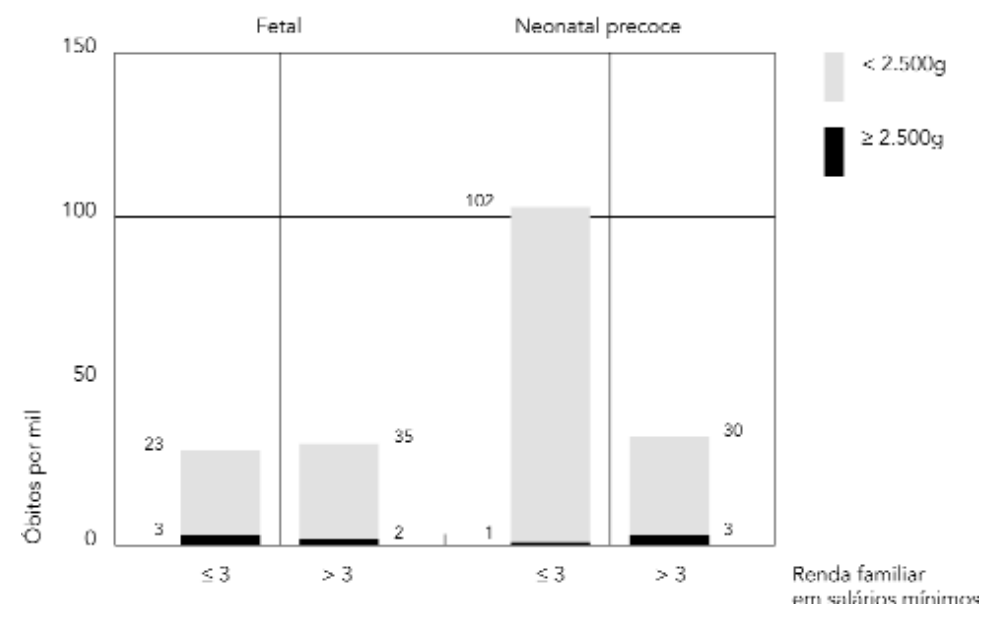

na assistência pré-natal, na atenção ao parto e ao recém-nascido. Esta última pode estar associada com a criação de três Unidades de Tratamento Intensivo Neonatal em Pelotas durante a década de 1980.

No que se refere à mortalidade fetal, a menor mortalidade nos óbitos antepartum possivelmente deve-se a melhores condições maternas e de atenção pré-natal, enquanto que o atendimento da gestante durante o trabalho de parto parece ainda apresentar algumas deficiências, tendo em vista que os índices de mortalidade fetal intrapartum permaneceram inalterados durante a década. A redução dos óbitos neonatais precoces no primeiro dia de vida 
pode ser devido a melhorias no atendimento intensivo oferecido pelas UTIs. Um achado inesperado é o aumento da mortalidade neonatal precoce no grupo de crianças entre 1.000 e 2.000 gramas em 1993, em relação a 1982, pois este grupo deveria beneficiar-se dos recursos proporcionados pelas UTIs. Razões para este fenômeno serão procuradas na avaliação cuidadosa das causas de óbito e do atendimento oferecido.

Embora toda uma estrutura tenha sido preparada para a realização de necrópsias, com a finalidade de um maior esclarecimento sobre a causa básica da morte, o percentual atingido foi de $31,7 \%$ para os óbitos neonatais precoces. Em razão da pouca aceitação dos familiares em relação às necrópsias, outras alternativas para a determinação da causa básica da morte precisam ser utilizadas. A classificação de Wigglesworth (Keeling et al., 1989) para a mortalidade perinatal tenta contornar a falta dos achados de autópsias na maioria dos estudos, agrupando em cinco categorias os óbitos perinatais conforme o peso ao nascer, momento do óbito em relação ao parto, idade gestacional e presença ou não de malformações congênitas. No presente estudo de 1993, houve um acréscimo importante de $39 \%$ para asfixia como principal causa de mortalidade perinatal. Novamente, este achado deve ser avaliado com cautela, le- vando em consideração a evolução das outras causas de óbito, lembrando que asfixia e imaturidade são causas de mortalidade perinatal que refletem a eficiência dos cuidados médicos (Clarke et al., 1993).

Outro achado que neste momento não pode ser explicado, mas que merece uma cuidadosa análise, é a menor mortalidade neonatal precoce observada entre crianças do sexo feminino.

Um fato importante demonstrado por este estudo é que, apesar da queda dos índices de mortalidade na década, as diferenças entre grupos sociais mantiveram-se muito grandes, pois enquanto o grupo de mais alta renda apresentou uma redução da mortalidade perinatal de 2,5 vezes nesta última década, a redução para crianças de famílias de baixa renda foi de uma vez e meia. Cabe salientar que a manutenção dos diferenciais de mortalidade perinatal conforme a renda, em 1993, deve-se ao componente neonatal precoce, e não à mortalidade fetal. É importante investigar por que os avanços tecnológicos ocorridos na década, que incluíram um aumento da assistência médica e a instalação de unidades de tratamento intensivo, não diminuíram as desigualdades sociais observadas nos indicadores de mortalidade de recém-nascidos. 


\section{Referências}

BARROS, F. C.; VICTORA, C. G.; TEIXEIRA, A. M. B. \& PUERTO FILHO, M., 1985. Mortalidade perinatal e infantil em Pelotas, Rio Grande do Sul: nossas estatísticas são confiáveis? Cadernos de Saúde Pública, 1:348-358.

CARTLIDGE, P. H. T. \& STEWART, J. H., 1995. Effect of changing the stillbirth definition onevaluationof perinatal mortality rates. The Lancet, 346:486488.

CLARKE, M.; MASON, E. S.; MACVICAR, J. \& CLAYTON, D. G., 1993. Evaluating perinatal mortality rates: effects of referral and case mix. British Medical Journal, 306:824-827.

DIS/ATMA/RS (Divisão de Informação em Saúde/Acessoria Técnica e do Meio Ambiente/Rio Grande do Sul), 1995. Estatística de Saúde: Mortalidade. Vol. 19. Porto Alegre: SSMA.

HORTA, B. L.; BARROS, F. C.; HALPERN, R. \& VICTORA, C. G.,1996. Baixo peso ao nascer em duas coortes de base populacional no Sul do Brasil: tendências e diferenciais. Cadernos de Saúde Pública, 12(supl. 1):27-31.

KEELING, J. W.; MAC GILLIVRA,Y. I.; GOLDING, J.; WIGGLESWORTH, J.; BERRY, J. \& DUNN, P. M., 1989. Classification of perinatal death. Archives of Disease in Childhood, 64:1345-1351.

MENEZES, A. M.; VICTORA, C. G.; BARROS, F. C.; ALVES, C.; ROCHA, C.; ALBERNAZ, E.; MENEZES, F. S. \& JANNKE, H. A., 1996. Mortalidade infantil em duas coortes de base populacional no Sul do Brasil: tendências e diferenciais. Cadernos de Saúde Pública, 12(supl. 1):79-86.
MORRIS, N. M.; UDERY, J. R. \& CHASE, C. L., 1975. Shifting age-parity distribution of births and the decrease in infant mortality. American Journal of Public Health, 65:359-362.

OMS (Organização Mundial da Saúde), 1980. Classificação Internacional de Doenças. Revisão 1975. São Paulo: Centro da OMS para Classificação de Doenças em Português.

TOMASI, E.; BARROS, F. C. \& VICTORA, C. G., 1996. As mães e suas gestações: comparação de duas coortes de base populacional no Sul do Brasil. Cadernos de Saúde Pública, 12(supl. 1):21-25.

VICTORA, C. G.; BARROS, F. C.; HALPERN, R.; MENEZES, A. M.; HORTA, B. L.; TOMASI, E.; WEIDERPASS, E.; CESAR, J. A.; OLINTO, M. T.; GUIMARÃES, P. R. V.; GARCIA, M. del M \& VAUGHAN, J. P. Estudo longitudinal da população maternoinfantil de Pelotas, RS, 1993: aspectos metodológicos e resultados preliminares. Revista de Saúde Pública.

VICTORA, C. G.; BARROS, F. C.; TOMASI, E.; MENEZES, A. M.; HORTA, B. L.;. WEIDERPASS, E.; CESAR, J. A.; OLINTO, M. T.; HALPERN, R.; GARCIA, M. del M. \& VAUGHAN, J. P., 1996. Tendências e diferenciais na saúde materno-infantil: delineamento e metodologia das coortes de 1982 e 1993 de mães e crianças de Pelotas, RS. Cadernos de Saúde Pública, 12(supl. 1):7-14.

WIGGLESWORTH, J. S., 1980. Monitoring perinatal mortality: a pathophysiological approach. The Lancet, 27:684-686. 